\title{
ON SUPERSOLVABILITY OF FINITE GROUPS
}

\author{
PIROSKA CSÖRGŐ \\ Eötvös University, Dept. of Algebra and Number Theory, Pàzmàny Pèter Sètàny $1 / c$, \\ H-1117 Budapest, Hungary \\ e-mail: skacs.elte.hu
}

(Received 21 June, 1999; accepted 28 March 2001)

\begin{abstract}
We prove a natural factorization of supersolvable groups and then we give another characterization of them in connection with the Fitting subgroup. Applying these theorems we describe the structure of some subclasses of supersolvable groups.
\end{abstract}

2000 Mathematics Subject Classification. 20D40, 20D10.

Introduction. All groups considered in this note are finite. Recall that a group $G$ is called supersolvable if its every chief factor is cyclic. We say following Kegel [1] that a subgroup of $G$ is $S$-quasinormal in $G$ if it permutes with every Sylow subgroup of $G$. $\pi(G)$ denotes the set of prime divisors of the order of the group $G$. Several authors examined the structure of a group under the assumption that some subgroups are well-situated in the group. They studied the influence of $S$-quasinormality of some subgroups of a finite group $G$ which ensures the supersolvability of $G$. In this paper we prove a natural factorization of supersolvable groups. The corollary of this theorem is another characterization of supersolvable groups based on the structure of the Fitting subgroup. By using these results we describe the structure of these subclasses of supersolvable groups obtained under the assumption that some subgroups satisfy certain conditions.

Main results. Huppert proved [2, Satz 10.3, p. 724] the following theorem. If a finite group is the product of pairwise permutable cyclic subgroups, then it is supersolvable. Of course the converse of this statement is not even true in the class of nilpotent groups, since there are nonabelian groups of exponent $p$ when $p>2$. By studying it, we find that a supersolvable group can be decomposed as a product of cyclic subgroups of prime power order that are permutable if their orders are powers of different primes and those belonging to the same prime satisfy certain conditions.

THeOREM 1. Let $G$ be a group with $\pi(G)=\left\{p_{1}, \ldots, p_{k}\right\}$. Then $G$ is supersolvable if and only if for all $p_{i} \in \pi(G)$ there is a Sylow $p_{i}$-subgroup $P_{i}$ and cyclic subgroups $P_{i_{l}}$ $\left(1 \leq l \leq t_{i}\right)$ of $P_{i}$ such that

(i) $P_{i}=P_{i_{1}} P_{i_{2}} \ldots P_{i_{t_{i}}}$,

(ii) $P_{i_{1}} \ldots P_{i_{l}} \triangleleft P_{i}$, for all $1 \leq l \leq t_{i}$

(iii) $P_{i_{l}} \cdot P_{j_{m}}=P_{j_{m}} \cdot P_{i_{l}}$, for all $1 \leq i, j \leq k, i \neq j, 1 \leq l \leq t_{i}, 1 \leq m \leq t_{j}$.

This paper was partly supported by Hungarian National Foundation for Scientific Research Grant \# T 029132 and T 022925. 
Moreover, for every chief series refining a Sylow tower there exists such a factorization of Sylow subgroups as given above.

For the proof we need the following result.

Lemma 1. Let $A$ be an abelian normal Sylow p-subgroup of a group $G$. Let a $\in A$ be of order $p$ such that $\langle a\rangle$ is normal in $G$ and $A /\langle a\rangle$ is cyclic. Then either $A$ is cyclic or $A=\langle a\rangle \times\langle b\rangle$, where $\langle b\rangle$ is normal in $G$.

Proof. Assume that $A$ is not cyclic. Obviously $G / A$ acts on $A / \Phi(A)$. By Maschke's Theorem $A / \Phi(A)=\langle a \Phi(A)\rangle \times\langle b \Phi(A)\rangle$, where $\langle b \Phi(A)\rangle$ is $G / A$-invariant. As $A$ is abelian, $A=\langle a\rangle\langle b\rangle$ holds. Since $|a|=p$ we conclude that here $|A:\langle b\rangle|=p$. Also $\langle b\rangle \supseteq \Phi(A)$, whence $\langle b\rangle$ is $G / A$-invariant. Consequently $\langle b\rangle \triangleleft G$.

Lemma 2. Let $G$ be a supersolvable group. Let $P$ be a normal Sylow p-subgroup of $G$ and $H$ a p-complement. Then $P=A_{1} \cdot A_{2} \ldots A_{s}$, where every $A_{i}$ is a cyclic subgroup of $P$ such that $H \leq N_{G}\left(A_{i}\right)$ and $A_{1} A_{2} \ldots A_{i} \triangleleft P$, for all $1 \leq i \leq s$.

Proof. We prove our statement by induction on the order of $P$. The supersolvability of $G$ implies that there exists a subgroup $A_{1}$ of $P$ of order $p$ such that $A_{1}$ is normal in $G$. We can assume that $A_{1} \neq P$. Obviously $G / A_{1}$ satisfies the conditions of our lemma. By induction on $G / A_{1}$ there exist cyclic subgroups $D_{2} / A_{1}, \ldots, D_{s} / A_{1}$ of $P / A_{1}$ such that $H A_{1} / A_{1} \leq N_{G / A_{1}}\left(D_{i} / A_{1}\right)$ and $\left(D_{2} / A_{1}\right) \ldots\left(D_{s} / A_{1}\right) \triangleleft P / A_{1}=$ $D_{2}\left(A_{1}\right) \ldots D_{s}\left(A_{1}\right)$, for all $2 \leq i \leq s$. Thus $H \leq N_{G}\left(D_{i}\right)$ and, since $A_{1} \leq Z(P)$, the $D_{i}$ are abelian. Applying Lemma 1 to $D_{i}$ and $A_{1}$, we can see that for all $i$ there is a cyclic subgroup $A_{i}$ of $D_{i}$ such that $D_{i}=A_{1} \cdot A_{i}$ and $A_{i}$ is normalized by $H$. The subgroups $A_{i}$ have the required properties.

Proof of Theorem 1. First we assume the supersolvability of $G$. Then $G$ possesses an ordered Sylow tower by [2, Satz 9.1, p. 716]. Suppose $p_{1}>p_{2}>\ldots>p_{k}$. Then for each $p_{i}$ we have a Sylow $p_{i}$-subgroup $P_{i}$ such that $P_{i} \leq N_{G}\left(P_{j}\right)$ for all $j<i$. Using the normality of $P_{i}$ in $P_{i} P_{i+1} \ldots P_{k}$ we apply Lemma 2 to $P_{i}$ and $P_{i} P_{i+1} \ldots P_{k}$. We get a factorization $P_{i}=P_{i_{1}} \ldots P_{i_{t_{i}}}$, where $P_{i_{r}}$ is a cyclic subgroup normalized by $P_{i+1} \ldots P_{k}$ and $P_{i_{1}} \ldots P_{i_{r}}$ is normal in $P_{i}$, for all $1 \leq r \leq t_{i}$. Let $1 \leq i, j \leq k, i \neq j$, $1 \leq l \leq t_{i}, 1 \leq m \leq t_{j}$. Assume $i<j$. Then, as stated before, $P_{i_{l}}$ is normalized by $P_{j}$, whence $P_{i_{l}} P_{j_{m}}=P_{j_{m}} P_{i_{l}}$ holds.

Conversely, assume that $G$ is a group satisfying (i), (ii), and (iii) of the Theorem. Suppose $p_{1}>p_{2}>\ldots>p_{k}$. Let $N=P_{11}$. By hypothesis we have $N \triangleleft P_{1}$ and $N P_{i_{l}}=P_{i_{l}} N$, for all $2 \leq i \leq k$ and $1 \leq l \leq t_{i}$. By Ito's Theorem [2, Satz 10.1, p. 722] we get that $N P_{i_{l}}$ is supersolvable whence, since $p_{1}>p_{i}, N \triangleleft N P_{i_{l}}$ follows. Therefore $P_{i} \leq N_{G}(N)$, for all $2 \leq i \leq k$. Thus we conclude $N \triangleleft G$. Since $G / N$ obviously inherits the hypothesis, we have by induction that $G / N$ is supersolvable. As $N$ is cyclic, we find $G$ is supersolvable.

M. Asaad and M. Ramadan in [3, Theorem 3.3] proved the following result. Suppose that $G$ is solvable and $\Phi(G)=1$. Then $G$ is supersolvable if and only if Fit $G$ is the direct product of some normal subgroups of $G$ of prime order.

Using our factorization on supersolvable groups we generalize the Theorem above. Not supposing $\Phi(G)=1$ we give another characterization of supersolvable groups. For this aim we introduce the following concept. 
Definition. A subgroup $H$ of $G$ is called weak $S$-quasinormal in $G$ if, for every $p \in \pi(G)$, there is at least one Sylow $p$-subgroup of $G$ that permutes with $H$.

REMARK. It follows from Theorem 1 that a supersolvable group is the product of some weak $S$-quasinormal cyclic subgroups of prime power orders.

THEOREM 2. For a group $G$ the following statements are equivalent.

(a) $G$ is supersolvable.

(b) $G^{\prime} \leq$ Fit $G$ and Fit $G$ is the product of cyclic and weak $S$-quasinormal subgroups of $G$ of prime power orders.

(c) There is a nilpotent normal subgroup $N$ of $G$, such that $G^{\prime} \leq N$ and $N$ is the product of cyclic and weak $S$-quasinormal subgroups of $G$ of prime power orders.

Proof. (a) $\Longrightarrow$ (b) Let $G$ be supersolvable. Then by [2, Satz 9.1, p. 716] $G^{\prime}$ is nilpotent, whence Fit $G \geq G^{\prime}$. The supersolvability implies the existence of an ordered Sylow tower. Let $\pi(G)=\left\{p_{1}, \ldots, p_{k}\right\}$ with $p_{1}>p_{2} \ldots>p_{k}$ and let $P_{1} P_{2} \ldots P_{i}(i=1, \ldots, k)$ be a Sylow tower of $G$. Clearly there exists a chief series refining our Sylow tower such that it contains $P_{1} P_{2} \ldots P_{i-1}\left(P_{i} \cap\right.$ Fit $\left.G\right)$ for all $1 \leq i \leq k$. Applying Theorem 1 , it is easy to see that $P_{i} \cap$ Fit $G$ is the product of weak $S$-quasinormal cyclic subgroups.

(b) $\Longrightarrow$ (c) This is trivial, because we may choose $N=$ Fit $G$.

(c) $\Longrightarrow$ (a) Hypothesis (c) is obviously inherited by all quotient groups. Let $G$ be a group of minimal order that is not supersolvable but satisfies (c). By the minimality we conclude that $G$ has a unique minimal normal subgroup $M$. As $G$ is solvable, $\Phi(G)=1$ and $M=$ Fit $G$. Obviously $N=M=G^{\prime}$ is an elementary abelian $p$ group, for some prime $p$, and $G / N$ is a $p^{\prime}$-group, so that $N$ is the Sylow $p$-subgroup of $G$. By the conditions $N=N_{1} \cdot N_{2} \cdot \ldots \cdot N_{t}$ with cyclic and weak $S$-quasinormal subgroup $N_{i}$ of $G$. The weak $S$-quasinormality implies that for every $q \neq p$ there is a Sylow $q$-subgroup $Q$ such that $Q N_{i}=N_{i} Q$ for all $1 \leq i \leq t$. Since $N_{i}$ is subnormal in $G$, obviously $N_{i} \triangleleft N_{i} Q$. As $N_{i} \triangleleft N$, we find $N_{G}\left(N_{i}\right)=G$, whence $N_{i}=N$. We have $N_{i}$ is cyclic and consequently $G$ is supersolvable, a contradiction.

We try to weaken these conditions to give another characterization of supersolvable groups.

Theorem 3. Let $G$ be a group with $G^{\prime} \leq$ Fit $G$. Then $G$ is supersolvable if and only if there exists a normal subgroup $H$ of $G$ such that $G / H$ is supersolvable and Fit $H$ is the product of cyclic and weak $S$-quasinormal subgroups of $G$.

Proof. (1) Assume that $G$ is supersolvable. We may choose $H=$ Fit $G$. Using our Theorem 2, we conclude that $H$ satisfies the conditions.

(2) Let $G$ be a group of minimal order that is not supersolvable, but has got a normal subgroup $H$ with the required properties. We now aim to show that $\Phi(G)=1$. Assume $\Phi(G) \neq 1$. Since Fit $G \geq G^{\prime}, G$ is solvable, whence Fit $G \neq \Phi(G)$. Clearly Fit $G \cap H=$ Fit $H$. If $\Phi(G) \cap H=$ Fit $G \cap H$, using again Fit $G \geq G^{\prime}$, we conclude that $H \Phi(G) / \Phi(G)$ is abelian and $H \Phi(G) / \Phi(G) \cap$ Fit $G / \Phi(G)=1$. We have $H \Phi(G) / \Phi(G) \triangleleft G / \Phi(G)$ and further $\operatorname{Fit}(G / \Phi(G))=$ Fit $G / \Phi(G)$. As $G$ is solvable, $C_{G / \Phi(G)}(\operatorname{Fit}(G / \Phi(G))) \leq \operatorname{Fit}(G / \Phi(G))$. It follows that $H \leq \Phi(G)$, whence $G / \Phi(G)$ is supersolvable. Using Huppert's Theorem [2, Satz VI.8.6] we get that $G$ is super- 
solvable, contradicting the minimality of $G$. Thus $\Phi(G) \cap H \neq$ Fit $G \cap H=$ Fit $H$. Obviously $G / \Phi(G)$ satisfies the conditions of our theorem. The minimality of $G$ yields the supersolvability of $G / \Phi(G)$. Using again Huppert's Theorem we find that $G$ is supersolvable, a contradiction. Thus $\Phi(G)=1$.

The supersolvability of $G / H$ implies the existence of the following chain: Fit $G \cap H=$ Fit $H=F_{0} \triangleleft F_{1} \triangleleft \ldots \triangleleft F_{k}=$ Fit $G$ such that $F_{i} \triangleleft G$ and $F_{i} / F_{i-1}$ is of prime order for all $1 \leq i \leq k$. Assume $F_{i} / F_{i-1}$ is of order $p$. Let $H$ be a Hall subgroup of $G$ with $\pi(H)=\pi(G) \backslash\{p\}$. Then $H$ acts on $F_{i}$ and $F_{i-1}$. Using Maschke's Theorem we get $F_{i}=F_{i-1} \times\left\langle b_{i}\right\rangle$ and $H \leq N_{G}\left(\left\langle b_{i}\right\rangle\right)$ so that $\left\langle b_{i}\right\rangle$ is weak $S$-quasinormal in $G$. As we have Fit $G \cap H$ is the product of cyclic weak $S$-quasinormal subgroups of $G$, we conclude that Fit $G$ is the product of cyclic weak $S$-quasinormal subgroups of $G$. Applying our Theorem 2 we find that $G$ is supersolvable. This is the final contradiction.

For the study of the structure of some subclasses of supersolvable groups we prove the following result.

THeORem 4. Let $G$ be a supersolvable group and $U$ a normal p-subgroup of $G$ with $p \neq 2$. Then every minimal subgroup of $U$ is normal in $U$ and $S$-quasinormal in $G$ if and only if there is a chain $1=U_{0} \triangleleft U_{1} \triangleleft \ldots \triangleleft U_{k}=U$ with $U_{i} \triangleleft G,\left|U_{i} / U_{i-1}\right|=p$ for every $1 \leq i \leq k$ and $\Omega_{1}(U)=U_{l} \leq Z(U)$, for some $1 \leq l \leq k$. Moreover, for every $g \in G$ with $(|g|, p)=1$, there exists a natural number $t_{g}$ with $1 \leq t_{g} \leq p-1$ such that $a^{g}=a^{t_{g}}$, where $a$ is an arbitrary element of $D=\stackrel{\underset{i=1}{\times}}{\times}\left(U_{i} / U_{i-1}\right)$.

Proof. Assume that every minimal subgroup of $U$ is normal in $U$ and $S$-quasinormal in $G$. Then these minimal subgroups are clearly in $Z(U)$; that is $\Omega_{1}(U) \leq Z(U)$. By the supersolvability of $G$ there is a chain $1=U_{0} \triangleleft U_{1} \triangleleft \ldots \triangleleft U_{k}=U$ such that $U_{i} \triangleleft G$ and $U_{i} / U_{i-1}$ is of order $p$, for all $1 \leq i \leq k$. Moreover $U_{l}=\Omega_{1}(U)$, for some $1 \leq l \leq k$. Let $g \in G$ with $g \neq 1,(|g|, p)=1$ and $u \in \Omega_{1}(U), u \neq 1$. As $\langle u\rangle$ is subnormal in $G$, and $g$ is the product of elements of prime power orders, the $S$-quasinormality implies $g \in N_{G}(\langle u\rangle)$. Thus we have $u^{g}=u^{t_{g}}$, for some natural number $t_{g}$ with $1 \leq t_{g} \leq p-1$. Let $v \in \Omega_{1}(U), 1 \neq v \neq u$. Similarly $v^{g}=v^{l}$ where $1 \leq l \leq p-1$. Since $(u v)^{g}=(u v)^{k}$, for some natural number $1 \leq k \leq p-1$, using $\Omega_{1}(U) \leq Z(U)$ we find $k=t_{g}=l$. Thus $w^{g}=w^{t_{g}}$, for all $w \in \Omega_{1}(U)$. Apply our factorization Theorem 1 to $U\langle g\rangle$ and to the chief series $1=U_{0} \triangleleft U_{1} \triangleleft \ldots \triangleleft U_{k}=U \triangleleft U\langle g\rangle$. Then for every $i \leq i \leq k$ there exists $a_{i} \in U_{i}$ such that $U_{i-1}\left\langle a_{i}\right\rangle=U_{i}$ and $g \in N_{G}\left(\left\langle a_{i}\right\rangle\right)$. Suppose $\left|a_{i}\right|=p^{m_{i}}$ and $a_{i}^{g}=a_{i}{ }^{t_{i}}$, for some natural number $1 \leq t_{i} \leq p^{m_{i}}-1$. Since $\left(a_{i}^{p^{m_{i}-1}}\right)^{g}=\left(a^{p^{m_{i}-1}}\right)^{t_{g}}$ we conclude $t_{i} \equiv t_{g}(p)$. Moreover $\bar{a}_{i}^{g}=\bar{a}_{i}^{t_{g}}$ is true for all $1 \leq i \leq k$ and $\bar{a}_{i} \in U_{i} / U_{i-1}$. Consequently $a^{g}=a^{t_{g}}$ follows for an arbitrary element $a$ of $D=\underset{i=1}{\stackrel{k}{\times}}\left(U_{i} / U_{i-1}\right)$.

Assume conversely that $G$ has got a chain with the required properties. As $\Omega_{1}(U) \leq Z(U)$, every minimal subgroup of $U$ is normal in $U$. Let $g \in G$ with $g \neq 1$, $(|g|, p)=1$. Applying Lemma 2 to $\Omega_{1}(U)\langle g\rangle$, we get $\Omega_{1}(U)=\left\langle b_{1}\right\rangle \times \ldots \times\left\langle b_{l}\right\rangle$ with $g \in N_{G}\left(\left\langle b_{i}\right\rangle\right)$ for all $i$. Using the hypothesis $b_{i}{ }^{g}=b_{i}{ }^{t_{g}}$ follows, whence $b^{g}=b^{t_{g}}$ holds for every $b \in \Omega_{1}(U)$. It easily follows from this fact that every minimal subgroup of $U$ is $S$-quasinormal in $G$.

In [4, Theorem 1] M. Asaad and the author have shown the following result. Let $\mathcal{F}$ be a saturated formation containing the class of supersolvable groups. Suppose 
that $G$ is a group with a normal subgroup $H$ such that $G / H \in \mathcal{F}$. If every subgroup of Fit $H$ of prime order or order 4 is $S$-quasinormal in $G$, then $G \in \mathcal{F}$. A corollary of this theorem [4, Corollary 4$]$ is the following result. If $G$ is solvable and every subgroup of Fit $G$ of prime order or order 4 is $S$-quasinormal in $G$, then $G$ is supersolvable. Examining the structure of this subclass of supersolvable groups we obtain the following result.

THeORem 5. Let $G$ be a solvable group. Then every subgroup of Fit $G$ of prime order or order 4 is $S$-quasinormal in $G$ if and only if $G=M(N \times K)$, where $M$ is a nilpotent normal subgroup of odd order, $N$ is a nilpotent subgroup, $K$ is a nilpotent Hall subgroup such that $M \cap(N \times K)=1$, Fit $G \cap N=1$ and every minimal subgroup of $M$ is normal in $M$ and $S$-quasinormal in $G$.

Thus we can apply our Theorem 4 to the Sylow subgroups of $M$, and we get a complete characterization.

For the proof we need the following results.

Lemma 3. Let $U$ be a 2-group in a group $G, a \in N_{G}(U)$ with $(|a|, 2)=1$ and $a$ normalizes every minimal subgroup of $U$ and every cyclic subgroup of order 4 . Then $a \in C_{G}(U)$.

Proof. Let $h$ be an element of $U$ of order 4. By the conditions either $h^{a}=h$ or $h^{a}=h^{3}$. If $h^{a}=h^{3}$, then $a^{2} \in C_{G}(h)$. Since $a$ is of odd order, we conclude $a \in C_{G}(h)$. Consequently $a \in C_{G}\left(\Omega_{2}(U)\right)$, which yields $a \in C_{G}(U)$.

Lemma 4. Let $P$ be a normal p-subgroup of a solvable group $G$ with an odd prime p. Suppose every minimal subgroup of $P$ is $S$-quasinormal in $G$. One of the following holds:

(1) every minimal subgroup of $P$ is normal in $P$,

(2) $Q \leq C_{G}(P)$ for every Sylow $q$-subgroup $Q$ of $G$ with $q \neq p$.

Proof. Assume there exists an element $x_{0}$ of $P$ such that $\left|x_{0}\right|=p$ and $\left\langle x_{0}\right\rangle$ is not normal in $P$. The solvability of $G$ implies the existence of a Hall subgroup $H$ with $\pi(H)=\pi(G) \backslash\{p\}$. As $P$ is normal in $G,\left\langle x_{0}\right\rangle$ is subnormal in $G$. From the $S$-quasinormality of $\left\langle x_{0}\right\rangle$ we easily conclude that $H \leq N_{G}\left(\left\langle x_{0}\right\rangle\right)$. Let $H_{1}$ be the normal closure of $H$ in $G$. Obviously $H_{1} \leq N_{G}\left(\left\langle x_{0}\right\rangle\right)$. As $\left\langle x_{0}\right\rangle$ is not normal in $G$, we find that $H_{1} \cap P=P_{0} \neq P$. Using $P \triangleleft H_{1} P$ and $H_{1} \triangleleft H_{1} P$ we have that the elements of $H$ fix the elements of $P / P_{0}$ by conjugation. Applying Glauberman's Theorem [5] we get that there exists $v \in P \backslash P_{0}$ such that $H \leq C_{G}(v)$. Clearly the elements of $H$ normalize every minimal subgroup of $P$. Applying [4, Lemma 4] $H \leq C_{G}(P)$ holds. Let $Q$ be a Sylow $q$-subgroup of $G$ with $q \neq p$. As $Q \leq H^{z}$ for some $z \in G$ and $P$ is normal in $G$, our statement follows.

Proof of Theorem 5. Suppose every subgroup of Fit $G$ of order prime or 4 is $S$-quasinormal in $G$. It follows from [4, Corollary 4] that $G$ is supersolvable, whence $G^{\prime}$ is nilpotent and $G^{\prime} \leq$ Fit $G$. Using [2, Satz 3.10 p. 271] $G=H$ Fit $G$ for some nilpotent subgroup $H$. If $P$ is an arbitrary Sylow subgroup of Fit $G$, denote by $P^{*}$ the unique Sylow subgroup of the subgroup $H P$ containing $P$. Denote by $\mathcal{S}$ the set of those Sylow subgroups $P$ of Fit $G$ for which $H P$ is nilpotent. Define $\mathcal{S}^{*}=\left\{P^{*} / P \in \mathcal{S}\right\}$. 
Suppose $P_{1}{ }^{*}, P_{2}{ }^{*} \in \mathcal{S}^{*}$. From the above we can easily conclude that $P_{1}{ }^{*} \leq C_{G}\left(P_{2}{ }^{*}\right)$. Let $K$ be the direct product of the elements of $\mathcal{S}^{*}$. We have Fit $G=M \times(K \cap$ Fit $G)$ and $H=N \times(H \cap K)$, for some nilpotent subgroup $N$ and a nilpotent Hall subgroup $M$. Let $B$ be the Sylow 2-subgroup of Fit $G$ and $h$ an arbitrary element of odd order of $H$. By the conditions, using Lemma $3, h \in C_{G}(B)$ holds, whence $B H$ is nilpotent; consequently $M$ is of odd order. We have $N K=N \times K, M \triangleleft G, N M \triangleleft G$, and so we find $G=M(N \times K)$. Assume $Q$ is a Sylow subgroup for an odd prime of Fit $G$ with $H \cap Q \neq 1$. Obviously $H=\left(H \cap Q^{*}\right) \times T$ for some Hall subgroup $T$ of $H$. Hence $T \leq C_{G}(H \cap Q)$ and $T \leq N_{G}(Q)$. By using our hypothesis and applying [4, Lemma 4] we get $T \leq C_{G}(Q)$. Thus in this case $Q^{*} \in \mathcal{S}^{*}$, that is $Q^{*} \leq K$. This fact implies $M \cap N=1$ and $M \cap(N \times K)=1$. Let $R$ be an arbitrary Sylow subgroup of $M$. By our Lemma 4 every minimal subgroup of $R$ is normal in $R$ and consequently in $M$ too, and is $S$-quasinormal in $G$. Obviously Fit $G \cap N=1$.

Assume conversely $G=M(N \times K)$ has the required properties. Clearly $M \leq$ Fit $G \leq M K$. Let $D$ be a subgroup of Fit $G$ of prime order or order 4. Supposing $D \leq M, D$ is $S$-quasinormal in $G$. If $D \leq K$, using the structure of $G$, it is easy to see the $S$-quasinormality of $D$ in $G$.

Asaad, Ramadan and Shaalan proved the following result [6, Corollary 4.3]. If $G$ is a solvable group and every maximal subgroup of any Sylow subgroup of Fit $G$ is $S$-quasinormal in $G$, then $G$ is supersolvable.

The study of the structure of this subclass of supersolvable groups has led to the following result.

Theorem 6. Let $G$ be a solvable group. Then every maximal subgroup of a Sylow subgroup of Fit $G$ is $S$-quasinormal in $G$ if and only if, for every Sylow p-subgroup $P$ of Fit $G$ and for every element $g$ of $G$ with $(|g|, p)=1$, there exists a natural number $t_{g}$ such that $1 \leq t_{g} \leq p-1$ and $\bar{a}^{g}=\bar{a}^{t_{g}}$ is true, for all $\bar{a} \in P / P \cap \Phi(G)$.

Proof. Assume every maximal subgroup of any Sylow subgroup of Fit $G$ is $S$-quasinormal in $G$. Then, by [6, Corollary 4.3], $G$ is supersolvable. Let $P$ be an arbitrary Sylow $p$-subgroup of Fit $G$ and $g \in G$ with $(|g|, p)=1$. Applying our Lemma 2 to $P$ and $P\langle g\rangle$, we find $P=A_{1} \ldots A_{k}$, where $A_{i}$ is a cyclic subgroup of $P$, normalized by $\langle g\rangle$ and $A_{1} A_{2} \ldots A_{i} \triangleleft P$, for all $1 \leq i \leq k$. We have $\Phi(P) \leq \Phi(G)$ and $P / P \cap \Phi(G)$ is an elementary abelian $p$-group. Consequently $P / P \cap \Phi(G)=$ $\tilde{P}=\tilde{A}_{1} \times \ldots \times \tilde{A}_{k}$, where $\tilde{A}_{i}=A_{i} / A_{i} \cap \Phi(G)$ and $\tilde{A}_{i}=\left\langle\bar{a}_{i}\right\rangle$. Obviously for every $1 \leq i \leq k$ there exists $1 \leq t_{i} \leq p-1$ such that $\bar{a}_{i}^{g}=\bar{a}_{i} t_{i}$. It will suffice to show that $t_{i}=t_{1}$, for all $1<i \leq k$. Suppose $t_{l} \neq t_{m}$, for some $1 \leq l, m \leq k, l \neq m$. Let $\tilde{A}$ be the product of every such $\tilde{A}_{i}$, where $i$ is different from $l$ and $m$. Let $\tilde{B}=\left\langle\bar{a}_{l} \bar{a}_{m}\right\rangle$. Clearly $\tilde{A} \tilde{B}$ is a maximal subgroup in $\tilde{P}$. It follows from the conditions that $g$ acts on $\tilde{P} / \tilde{A} \tilde{B}$. As $\tilde{P}=\tilde{A} \tilde{B} \cdot \tilde{A}_{l}=\tilde{A} \tilde{B} \cdot \tilde{A}_{m}$ and $\bar{a}_{l}^{g}=\bar{a}_{l}^{t_{l}}$ and $\bar{a}_{m}^{g}=\bar{a}_{m}^{t_{m}}$, we find $t_{l}=t_{m}$, a contradiction. Putting $t_{g}=t_{1}$, this part of our Theorem follows.

The converse of this theorem is trivial.

\section{REFERENCES}

1. O. H. Kegel, Sylow Gruppen und Subnormalteiler endlicher Gruppen, Math. Z. 78 (1962), 205-221.

2. B. Huppert, Endliche Gruppen I (Springer-Verlag, 1979). 
3. M. Asaad and M. Ramadan, On the intersection of maximal subgroups of a finite group, Archiv Math. (Basel) 61 (1993), 206-214.

4. M. Asaad and P. Csörgő, The influence of minimal subgroups on the structure of finite groups, Archiv Math. (Basel), 72 (1999), 401-404. 125 .

5. G. G. Glauberman, Fixed points in group with operators, Math. Z. 84 (1964), 120

6. M. Asaad, M. Ramadan and A. Shaalan, Influence of $\pi$-quasinormality on maximal subgroups of Fitting subgroup of a finite group, Archiv Math. (Basel) 56 (1991), 521-527. 